\title{
Solving Linear Time Varying Systems by Orthonormal Bernstein Polynomials
}

\author{
Mahmood Dadkhah \\ Department of Mathematics, PayameNoor University, Tehran, Iran
}

Email address:

m_dadkhah@pnu.ac.ir

To cite this article:

Mahmood Dadkhah. Solving Linear Time Varying Systems by Orthonormal Bernstein Polynomials. Science Journal of Applied Mathematics and Statistics. Vol. 3, No. 4, 2015, pp. 194-198. doi: 10.11648/j.sjams.20150304.15

\begin{abstract}
In this paper we present a method to find the solution of time-varying systems using orthonormal Bernstein polynomials. The method is based upon expanding various time functions in the system as their truncated orthonormal Bernstein polynomials. Operational matrix of integration is presented and is utilized to reduce the solution of time-varying systems to the solution of algebraic equations. Illustrative examples are included to demonstrate the validity and applicability of the technique.
\end{abstract}

Keywords: Orthonormal Bernstein Polynomials, Time Varying System, Operational Matrix, Linear Systems

\section{Introduction}

The history of orthogonal polynomials is very old. The Legendre polynomials originated from determining the force of attraction exerted by solids of revolution and their orthogonal properties were established by Adrien Marie Legendre during (1784-90). The problem of solving ordinary differential equations over infinite or semi-infinite intervals and of obtaining expansion of arbitrary functions over such intervals attracted the attention of famous mathematicians in the nineteenth century and to resolve it, functions known today as Hermite polynomials were introduced in 1864 by Charles Hermite (1822-1905).

In 1807, Joseph Fourier (1768-1830) while solving the partial differential equation encountered in connection with conduction of heat in a rod discovered that the solution can be expressed as a series of exponentially weighted sine functions. Later, he extended this idea to represent any arbitrary function as an infinite sum of sine and cosine functions.

Orthogonal functions (OFs) and polynomial series have received considerable attentions in dealing with various problems of dynamic systems and many papers have been written in various field of their application. The main approach is that of converting the underlying differential equation governing the dynamical systems to an algebraic form through the use of an operational matrix of integration $P$. The matrix $P$ can be uniquely determined based on the particular OFs. Special attentions has been given to applications of Walsh functions[6], block-pulse functions
[1,13,14], Laguerre polynomials [5], Legendre Polynomials [2,3], Chebyshev polynomials [4,10], Taylor series [9] and Fourier series $[7,11]$. The available sets of OFs can be divided into three classes. The first includes a set of piecewise constant basis functions (PCBFs) (e.g. Walsh, block-pulse, etc.). The second consists of a set of orthogonal polynomials (OPs) (e.g. Laguerre, Legendre, Chebyshev, etc). The third is the widely used set of sine-cosine functions (SCFs) in the form of Fourier series. In this paper we use orthonormal Bernstein polynomials to solve time varying systems.

The method reduces the original problem to solving linear algebraic equations. Therefore, computational difficulties are greatly reduced namely the method consists of reducing the problem to a set of algebraic equations by first expanding the candidate function as an orthonormal Bernstein polynomials with unknown coefficients. These orthonormal Bernstein polynomials are first introduced. The operational matrices of integration are given. This matrix is then used to evaluate the coefficients of the orthonormal Bernstein polynomials for the solution of time varying systems.

\section{Bernstein Polynomials and Their Properties}

The $B$-polynomial of $m$-th degree are defined on the interval $[0,1]$ as $([8,12])$

$$
B_{i, m}(t)=\left(\begin{array}{c}
m \\
i
\end{array}\right) t^{i}(1-t)^{m-i}, \quad 0 \leq i \leq m
$$


Where

$$
\left(\begin{array}{c}
m \\
i
\end{array}\right)=\frac{m !}{i !(m-i) !}
$$

Bernstein polynomials have some properties that distinguish it from other polynomials for example continuity and partition of unity property. All of the Bernstein polynomials vanish at the initial and end points of the interval $[a, b]$ except for the first polynomial and the last polynomial are equal to 1 at $x=a$ and $x=b$, respectively, which provides greater flexibility to impose boundary conditions. The Bernstein polynomials, although not based on orthogonal polynomials, can also be applied to analyze various problems. Specifically, Bernstein polynomials have been used for solving the partial differential equation.

It can be shown that each of the $B$-polynomials is positive and also the sum of all the $B$-polynomials is unity for all real $t$ belonging to the interval $[0,1]$, that is, $\sum_{i=0}^{m} B_{i, m}(t)=1$. Also it can be easily shown that any given polynomial of degree $m$ can be expanded in terms of linear combination of the basis functions

$$
\begin{aligned}
& O B[0,8](t)=\sqrt{(17)}(-1+t)^{8}, \\
& O B[1,8](t)=-\sqrt{(15)(17 t-1)(-1+t)^{7},} \\
& O B[2,8](t)=\sqrt{(13)}\left(136 t^{2}-32 t+1\right)(-1+t)^{6},
\end{aligned}
$$

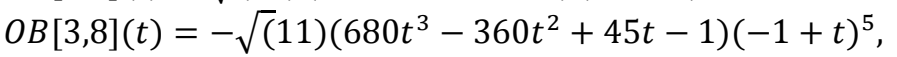

$$
\begin{aligned}
& O B[4,8](t)=\sqrt{(9)}\left(2380 t^{4}-2240 t^{3}+630 t^{2}-56 t+1\right)(-1+t)^{4}, \\
& O B[5,8](t)=-\sqrt{(7)}\left(6188 t^{5}-9100 t^{4}+4550 t^{3}-910 t^{2}+65 t-1\right)(-1+t)^{3} \text {, } \\
& O B[6,8](t)=\sqrt{(5)}\left(12376 t^{6}-26208 t^{5}+20475 t^{4}-7280 t^{3}+1170 t^{2}-72 t+1\right)(-1+t)^{2}, \\
& O B[7,8](t)=-\sqrt{(3)}\left(19448 t^{7}-56056 t^{6}+63063 t^{5}-35035 t^{4}+10010 t^{3}-1386 t^{2}+77 t-1\right)(-1+t) \text {, } \\
& O B[8,8](t)=24310 t^{8}-91520 t^{7}+140140 t^{6}-112112 t^{5}+50050 t^{4}-12320 t^{3}+1540 t^{2}-80 t+1 .
\end{aligned}
$$

Figure 1 shows these orthonormal functions.

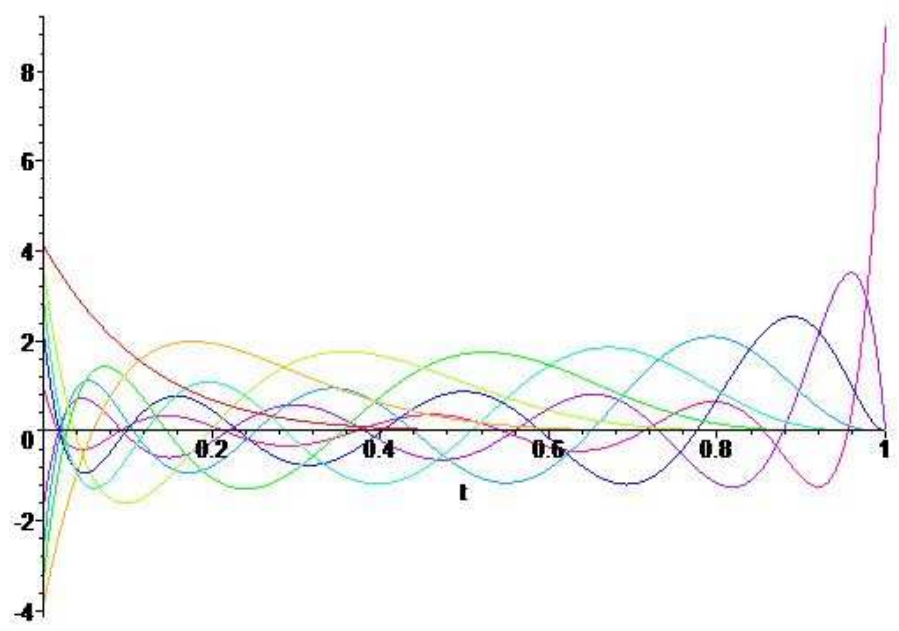

Figure 1. Orthonormal Bernstein olynomials with $m=8$

A function $f(t)$ belongs to the apace $L^{2}[0, L]$ may be expanded by Bernstein orthonormal polynomials as follows $[8,13]$ :

$$
f(t)=\sum_{i=0}^{\infty} C_{i m} O B_{i m}(t),
$$

where if $\langle$.$\rangle be the standard inner product on L^{2}[0,1]$ then

$$
C_{i m}=<f . O B_{i m}>.
$$

By truncating the series $(4)$ up to $(m+1)$ th term we can 
abtain an approximation for $f(t)$ as follows:

$$
f(t) \approx \sum_{i=0}^{m} C_{i m} O B_{i m}(t)=C^{T} B(t) .
$$

Where

$$
B(t)=\left[O B_{0, m}(t), O B_{1, m}(t), \ldots, O B_{m, m}(t)\right]^{T} .
$$

It can be easily seen that the elements of $B(t)$ in interval $[0, L]$ are orthogonal.

\section{Operational Matrix of Integration for Orthonormal Bernstein Polynomials}

In this section, we present the operational matrix of Integration for Bernstein orthonormal polynomials. To do this, first we introduce the concept of the operational matrix.

The operational properties of the orthogonal series may be written in terms of operational matrices of integration and differentiation. The main concept around these properties is the fact that the integral of an orthogonal series may be also expressed as a orthogonal series. The same can be stated for orthogonal series and their derivatives. In general terms the operational matrix of integration and differentiation may by defined as

$$
\begin{gathered}
\int_{0}^{t} \ldots \int_{0}^{t} \phi(s) d s=P^{n} \phi(t), \\
\frac{d^{n} \phi(t)}{d t^{n}}=D^{n} \phi(t) .
\end{gathered}
$$

Note that $\mathrm{P}$ and $\mathrm{D}$ are operational matrices of integration and differentiation respectively. Any given differential equation of degree $\mathrm{n}$ can be expressed as a set of state-space equations. This same set can be transformed into an algebraic set of equations using operational matrices.

Definition 1. Suppose

$$
\phi=\left[\phi_{0}, \phi_{1}, \ldots, \phi_{n}\right],
$$

where $\phi_{0}, \phi_{1}, \ldots, \phi_{n}$ are the basis functions on the given interval $[a, b]$. The matrices $Q_{n \times n}$ and $P_{n \times n}$ are named as the operational matrices of derivatives and integration

$$
P=\left[\begin{array}{ccc}
0.13265 & 0.25343 & 0.21934 \\
-0.009386 & 0.11224 & 0.21998 \\
0.0014151 & -0.016921 & 0.091837 \\
-0.0003403 & 0.0040701 & -0.022089 \\
0.00011506 & -0.0013759 & 0.0074675 \\
-0.0000495 & 0.00059211 & -0.003213 \\
0.00002144 & -0.000256 & 0.0013915
\end{array}\right.
$$

\section{Illustrative Examples}

In this section two examples are given to demonstrate the applicability, efficiency and accuracy of our proposed method. The following examples show the computational power of the Bernstein polynomial operational matrix of integration. respectively if and only if

$$
\begin{aligned}
\frac{d}{d t} \phi(t) & \approx Q \phi(t) \\
\int_{a}^{t} \phi(s) d s & \approx P \phi(t) .
\end{aligned}
$$

Operational matrices are used in several areas of numerical analysis and they hold particular importance in various subjects such as integral equations, differential and partial differential equations, optimal control and etc. Also many textbooks and papers have employed the operational matrices for spectral methods.

Using the operational matrix of an orthonormal system of functions to perform integration for solving, identifying and optimizing a linear dynamic system has several advantages:

(i) The method is computer oriented, thus solving higher order differential equation becomes a matter of dimension increasing,

(ii) The solution is a multi-resolution type

(iii) The solution is convergent, even though the size of increment may be large.

Until now, the operational matrix of integration has been determined for several types of orthogonal basis functions, such as the Walsh function, block- pulse function, Laguerre series, Chebyshev polynomials, Legendre polynomials and Fourier series.

The aim of present paper is to derive the Bernstein orthonormal polynomials matrix of integration namely $P_{m+1}$. The matrix $P_{m+1}$ may be used to solve problems of system analysis and synthesis in a manner similar to those of the other orthogonal functions. The Bernstein polynomials are first orthonormalized and the operational matrix of integration is then derived. With the same procedure as in [12], where the operational matrix of integration for Bernstein polynomials is derived, the integration of $B(t)$ in (5) can be approximated by $B(t)$ as follows:

$$
\int_{0}^{t} B(s) d s \approx P B(t) .
$$

Where $P$ is the operational matrix of integration of order $(m+1) \times(m+1)$ and with $m=6$ is given by:

$\left.\begin{array}{cccc}0.19503 & 0.16443 & 0.1275 & 0.07356 \\ 0.17501 & 0.15273 & 0.1166 & 0.06794 \\ 0.18408 & 0.12944 & 0.1092 & 0.05983 \\ 0.071429 & 0.14489 & 0.0831 & 0.05849 \\ -0.02414 & 0.051020 & 0.1010 & 0.03612 \\ 0.010392 & -0.02195 & 0.03061 & 0.04860 \\ -0.004499 & 0.009507 & -0.01325 & 0.01020\end{array}\right]$

\subsection{Example 1}

Consider a linear time-varying system

$$
\begin{aligned}
& y^{\prime}(t)+y(t)=1, \\
& y(0)=2,
\end{aligned}
$$


The exact solution is $1+e^{-t}$. Here we obtain approximated solution of (7) using the Bernstein operational matrix of integration $P$ with $m=6$ and compare the solutions. By Integration (7) from 0 to $t$, we get

$$
\begin{gathered}
\int_{0}^{t} y^{\prime}(s) d s+\int_{0}^{t} y(s)=\int_{0}^{t} 1 d s \\
\Rightarrow y(t)-y(0)+\int_{0}^{t} C^{T} B(s) d s=t \\
\Rightarrow C^{T} B(t)-C_{0}^{T} B(t)+C^{T} P B(t)=C_{1}^{T} B(t) .
\end{gathered}
$$

By simplifying we get

$$
C=\left(C_{0}+C_{1}\right)(I+P)^{-1} .
$$

Now $C$ can be computed. By substituting $C$ in (5), Figure 2 depicts the corresponding errors between the approximate solution and exact one.

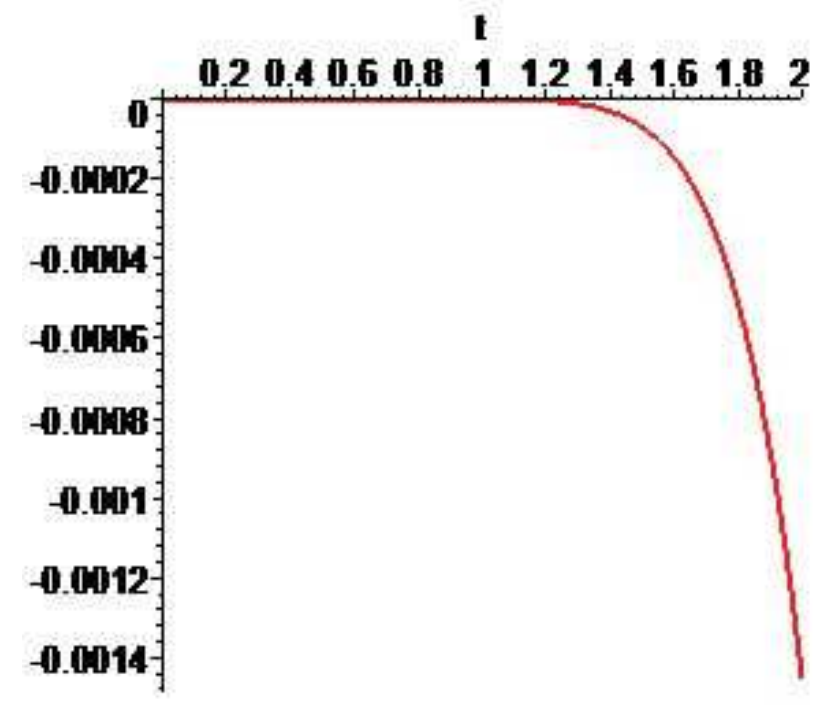

Figure 2. Corresponding error between the approximated solution and exact one in Example 1 with $m=6$

\subsection{Example 2}

Consider a linear time-varying system

$$
\begin{gathered}
y^{\prime}(t)-5 y(t)=4 t, \\
y(0)=0.25,
\end{gathered}
$$

The exact solution is $-4 / 25-(4 / 5) t+(41 / 100) e^{5 t}$. Here we obtain approximate solution of (8) using the Bernstein operational matrix of integration $P$ by taking $m=8$ and compare the solutions. Integrating (8) from 0 to $t$, we get

$$
\begin{aligned}
& \int_{0}^{t} y^{\prime}(s) d s-\int_{0}^{t} 5 y(s)=\int_{0}^{t} 4 s d s \\
& \Longrightarrow y(t)-y(0)-5 \int_{0}^{t} C^{T} B(s) d s=2 t^{2}
\end{aligned}
$$

$$
\Rightarrow C^{T} B(t)-C_{0}^{T} B(t)-5 C^{T} P B(t)=2 C_{1}^{T} B(t)
$$

By simplifying we get

$$
C=\left(C_{0}+2 C_{1}\right)(I-5 P)^{-1} .
$$

Now $C$ can be computed. By substituting $C$ in (5), Figure 3 depicts the corresponding errors between the approximate solution and exact one.

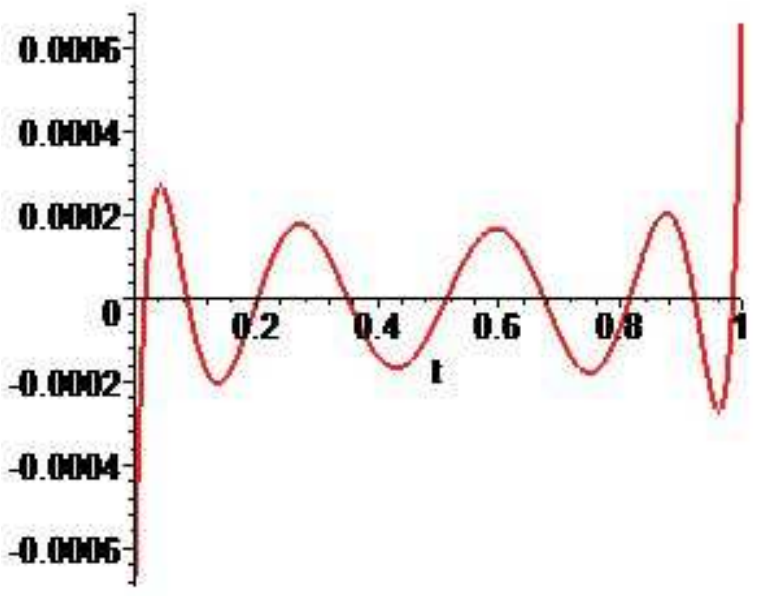

Figure 3. Corresponding error between the approximated solution and exact one in Example 2 with $m=8$

\section{Conclusion}

Using orthonormal Bernstein polynomials, a simple and computational method for solving linear time varying systems is considered. The method is based upon reducing a linear time varying systems to an algebraic linear or nonlinear problem. The unity of the function of orthogonality for Bernstein polynomials and the simplicity of applying Bernstein polynomials are great merits that make the approach very attractive and easy to use.

Although the method is simple, by solving various examples, accuracy in comparison of the other methods can be found. Furthermore this method can be extended to high order differential equations by appropriate changes.

\section{References}

[1] A. Deb, A. Dasgupta, G. Sarkar, A new set of orthogonal functions and its application to the analysis of dynamic systems, J. Frank. Inst. 343, 1-26, 2006.

[2] F. Khellat, S. A. Yousefi, The linear Legendre wavelets operational matrix of integration and its application, J. Frank. Inst. 343, 181-190, 2006.

[3] F. Marcellan, and W.V. Assche, Orthogonal Polynomials and Special Functions (a Computation and Appli- cations), Springer-Verlag Berlin Heidelberg, 2006. 
[4] H. R. Marzban and M. Shahsiah, Solution of piecewise constant delay systems using hybrid of block- pulse and Chebyshev polynomials, Optim. Contr. Appl. Met., Vol. 32, pp. 647-659, 2011.

[5] Kung, F. C. and Lee, H., Solution and parameter estimation of linear time-invariant delay systems using Laguerre polynomial expansion, Journal on Dynamic Systems, Measurement, and Control, 297-301, 1983.

[6] LÃ izaro I, Anzurez J, Roman M, Parameter estimation of linear systems based on walsh series, The Electronics, Robotics and Automotive Mechanics Conference, 355-360, 2009.

[7] M. H. Farahi, M. Dadkhah, Solving Nonlinear Time Delay Control Systems by Fourier series, Int. Journal of Engineering Research and Applications, Vol. 4, Issue 6, 217-226, 2014.

[8] M. I. Bhatti and P. Bracken, Solutions of differential equations in a Bernstein polynomial basis, Journal of Computational and Applied Mathematics, 205, 272 -280, 2007.

[9] M. Sezer, and A.A. Dascioglu, Taylor polynomial solutions of general linear differential-difference equations with variable coefficients, Appl. Math.Comput.174, 1526-1538, 2006.

[10] M. Shaban and S. Kazem and J. A. Rad, A modification of the homotopy analysis method based on Chebyshev operational matrices, Math. Comput. Model, in press(2013)

[11] Richard A. Bernatz. Fourier series and numerical methods for partial defferential equations. John Wiley \& Sons, Inc., New York, 2010.

[12] S. A. Youseï , M. Behroozifar, Operational matrices of Bernstein polynomials and their applications, Int. J. Syst. Sci. 41, 709-716, 2010.

[13] Wang, X., T, Numerical solutions of optimal control for time delay systems by hybrid of block-pulse functions and Legendre polynomials Applied Mathematics and Computation, 184, 849-856, 2007.

[14] Z.H. Jiang, W. Schaufelberger, Block Pulse Functions and Their Applications in Control Systems, Springer-Verlag, 1992. 